\title{
Area Preserving Brain Mapping
}

\author{
Zhengyu Su${ }^{1}$, Wei Zeng ${ }^{2}$, Rui Shi ${ }^{1}$, Yalin Wang ${ }^{3}$, Jian Sun $^{4}$, and Xianfeng $\mathrm{Gu}^{1}$ \\ ${ }^{1}$ Department of Computer Science, Stony Brook University \\ ${ }^{2}$ School of Computing and Information Sciences, Florida International University \\ ${ }^{3}$ School of Computing, Informatics, and Decision Systems Engineering, Arizona State University \\ ${ }^{4}$ Mathematical Sciences Center, Tsinghua University \\ \{zhsu,rshi,gu\}@cs.stonybrook.edu, wzeng@cis.fiu.edu, ylwang@asu.edu, sunjian0813@gmail.com
}

\begin{abstract}
Brain mapping transforms the brain cortical surface to canonical planar domains, which plays a fundamental role in morphological study. Most existing brain mapping methods are based on angle preserving maps, which may introduce large area distortions. This work proposes an area preserving brain mapping method based on MongeBrenier theory. The brain mapping is intrinsic to the Riemannian metric, unique, and diffeomorphic. The computation is equivalent to convex energy minimization and power Voronoi diagram construction. Comparing to the existing approaches based on Monge-Kantorovich theory, the proposed one greatly reduces the complexity (from $n^{2}$ unknowns to $n$ ), and improves the simplicity and efficiency.

Experimental results on caudate nucleus surface mapping and cortical surface mapping demonstrate the efficacy and efficiency of the proposed method. Conventional methods for caudate nucleus surface mapping may suffer from numerical instability; in contrast, current method produces diffeomorpic mappings stably. In the study of cortical surface classification for recognition of Alzheimer's Disease, the proposed method outperforms some other morphometry features.
\end{abstract}

\section{Introduction}

Nowadays surface parameterization has been used for a wide variety of applications like pattern recognition and medical imaging. Many prominent approaches, such as conformal mapping [18] and Ricci Flow [20] which have been employed to shape analysis [27, 7] and surface registration [35]. However, an accurate isometric parameterization is impossible for general surfaces.

The conformal mapping may bring huge area distortions in certain surfaces, e.g. a slim surface of brain caudate nucleus. In turn, such distortions usually introduce much difficulty for following shape analysis. As the clinical questions of interest move towards identifying very early signs of diseases, the corresponding statistical differences at the group level invariably become weaker and increasingly harder to identify. A stable method to compute some other mapping with alternative invariants may be highly advantageous for visualization and shape analysis in this research area.

In this work, we propose a novel method to compute area preserving mapping between surfaces. The mapping is diffeomorphic and unique under normalization. Moreover, the mapping is invariant under isometric transformations. We tested our algorithm on cortical and caudate nucleus surfaces extracted from 3D anatomical brain magnetic resonance imaging (MRI) scans. Figure 1 demonstrates the unique power that the area preserving mapping provides for brain cortical surface visualization when compared with its counterpart conformal mapping result. On cortical surfaces, the area preserving may also provide good visualization function to visualize those deeply buried sulci areas which otherwise are usually visualized with big area distortions. In a classification study, our algorithm achieved $87.50 \% \pm 0.55 \%$ average recognition rate with $95 \%$ confidence interval in a brain image dataset consisting of images of 50 healthy control (CTL) subjects and 50 Alzheimer's Disease (AD) patients. We also show that this novel and simple method can outperform two other morphometry features in the same dataset.

\subsection{Comparison}

The area preserving mapping is based on Optimal Mass Transport (OMT) theory, which has been applied for image registration and warping $[19,26]$ and visualization [36]. Our method has fundamental differences from these existing methods. 


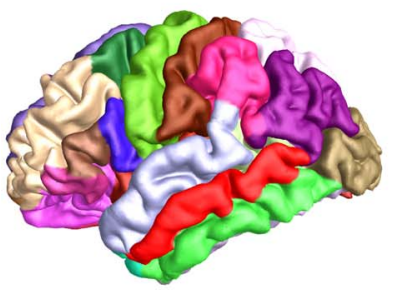

(a)

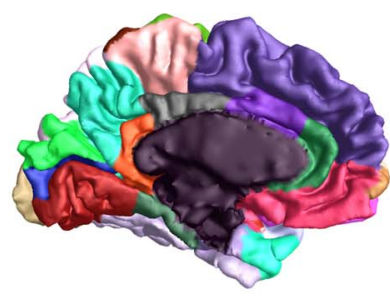

(b)

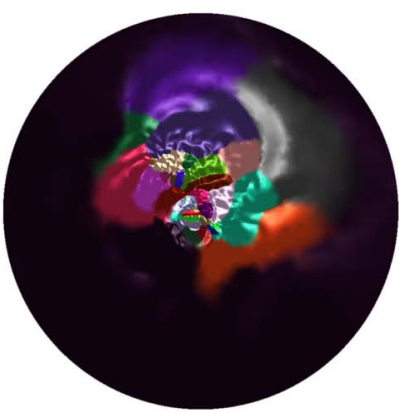

(c)

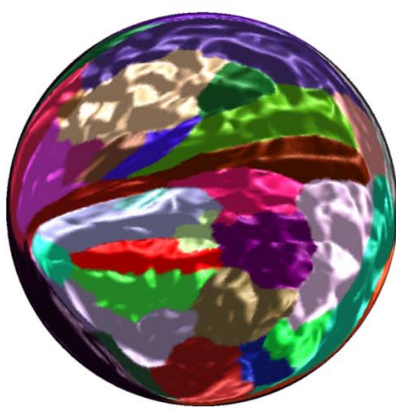

(d)

Figure 1. Comparison of geometric mappings for a left brain cortical surface: (a) brain cortical surface lateral view; (b) brain cortical surface medial view; brains are color coded according to functional area definition in [11]; (c) conformal mapping result; (d) area preserving mapping result. The results show that conformal mapping has much more area distortions on the areas close to the boundary while the area preserving mapping provides a map which preserves the area everywhere.

Monge considered the transportation cost for moving a pile of dirt from one spot to the other, then formulated the Optimal Mass Transport problem. Let $\Omega_{k} \subset \mathbb{R}^{n}$ be subdomains in $\mathbb{R}^{n}$, with positive density functions $\mu_{k}$, such that

$$
\int_{\Omega_{0}} \mu_{0} d x=\int_{\Omega_{1}} \mu_{1} d x
$$

Consider a diffeomorphism $f: \Omega_{0} \rightarrow \Omega_{1}$, which is mass preservation

$$
\mu_{0}=\left|J_{f}\right| \mu_{1} \circ f
$$

where $J_{f}$ is the Jacobian of the mapping $f$. The mass transport cost is

$$
C(f):=\int_{\Omega_{0}}|x-f(x)|^{2} \mu_{0}(x) d x .
$$

An optimal mass transport map, when it exists, minimizes the mass transport cost. There are two different approaches to prove the existence of the optimal mass transport map, i.e. Kantorovich's and Brenier's. Existing methods follow Kantorovich's approach [24], our proposed method follows Brenier's [8].

Kantorovich constructed a measure $\mu(x, y): \Omega_{0} \times \Omega_{1} \rightarrow$ $\mathbb{R}$, which minimizes the cost

$$
\int_{\Omega_{0} \times \Omega_{1}}|x-y|^{2} \mu(x, y) d x d y
$$

with the constraints

$$
\int_{\Omega_{1}} \mu(x, y) d y=\mu_{0}(x), \int_{\Omega_{0}} \mu(x, y) d x=\mu_{1}(y) .
$$

In contrast, Brenier showed there exists a convex function $u: \Omega_{0} \rightarrow \mathbb{R}$, such that its gradient map $\nabla u$ gives the optimal mass transport map, and preserves the mass:

$$
\mu_{0}=\operatorname{det}|H(u)| \mu_{1} \circ \nabla u .
$$

Conventional methods discretize each $\Omega_{k}$ to $n$ samples with discrete measures, and model the measure $\mu$ to an $n \times n$ matrix with linear constraints Eqn.2, such as a doublystochastic matrix (sum of each row and the sum of each column equal to one). The optimization of energy Eqn.1 is converted to a linear programming problem with $n^{2}$ variables.

In our current method, we only discretize the target domain $\Omega_{1}$ to $n$ points, then determine $n$ power weights for them, so that the power Voronoi diagram induced by the points and their power weights gives the optimal mass transport map. Furthermore, the $n$ power weights can be obtained by optimizing a convex energy.

Comparing to Kantorovich's approach, Brenier's approach has the following merits from computational point of view:

1. Complexity: Existing method has $n^{2}$ unknowns, whereas ours has only $n$ variables.

2. Uniqueness: Due to the convexity of the energy, our method has a unique solution.

3. Diffeomorphism: If the domains are convex, The optimal mapping is guaranteed to be diffeomorphic.

4. Efficiency: Due to the convexity of the energy, it can be optimized using Newton's method.

5. Simplicity: The computational algorithm is mainly based on (power) Voronoi diagram and Delaunay triangulation.

Furthermore, the obtained area preserving mapping between two surfaces is solely determined by the surface Riemannian metric, therefore it is intrinsic.

\subsection{Contributions}

Our major contributions in this work include: a way to compute area preserving mapping between surfaces based on Brenier's approach in Optimal Mass Transport theory. The current approach produces the unique diffeomorphic mapping. Comparing to the exiting method, the new 
method greatly reduces the complexity (from $n^{2}$ to $n$ ) and improves the simplicity and efficiency.

Thus our method offers a stable way to calculate area preserving mapping in $2 D$ parametric coordinates. To the best of our knowledge, it is the first work to compute area preserving mapping between surfaces based on Brenier's approach in OMT and apply it to map the profile of differences in surface morphology between healthy control subjects and $\mathrm{AD}$ patients. Our experimental results show our work may provide novel ways for shape analysis and improve the statistical detection power for detecting abnormalities in brain surface morphology.

\subsection{Related Work}

Conformal mapping and quasi-isometric embedding has been applied in computer vision for modeling the 2D shape space or 3D shape analysis [27, 9, 7]. Quasi-isometric brain parameterization has been investigated in $[13,6,12,31]$. Conformal brain mapping methods have been well developed in the field, such as circle packing based method in [21], finite element method [3, 23, 32], spherical harmonic map method [17], holomorphic differential method [33] and Ricci flow method [34].

Area preserving mapping has been applied for visualizing branched vessels and intestinal tracts in [36], which combined Kantorovich's approach with conformal mapping. Optimal mass transport mapping based on Kantorovich's approach has been applied for image registration in [19]. An improved multi-grid version of OMT mapping is presented in [26]. Comparing to the existing method, our method is based on Monge-Brenier theory to compute the Optimal Transport mapping and achieves the area preserving.

\section{Theoretic Foundation}

Power Diagram Consider a collection of points $P=$ $\left\{p_{1}, p_{2}, \cdots, p_{k}\right\}$ in $\mathbb{R}^{n}$. Suppose each point $p_{i} \in P$ has a power (weight) $h_{i} \in \mathbb{R}$. The power distance from a point $x \in \mathbb{R}^{n}$ to $p_{i}$ is defined as

$$
\operatorname{Pow}\left(x, p_{i}\right):=\left|x-p_{i}\right|^{2}-h_{i} .
$$

The power diagram of $\left\{\left(p_{i}, h_{i}\right)\right\}$ is a partition of the $\mathbb{R}^{n}$ into $k$ cells $W_{i}$, such that a point $x$ belongs to $W_{i}$ whenever

$$
\operatorname{Pow}\left(x, p_{i}\right)=\min _{j} \operatorname{Pow}\left(x, p_{j}\right) .
$$

We denote the area of $W_{i}$ as $w_{i}$, call it the area weight. The dual graph of the power diagram is called the power Delaunay triangulation.

For each site $\left(p_{i}, h_{i}\right)$, we define the supporting hyperplane

$$
x_{n+1}=x^{T} p_{i}-p_{i}^{T} p_{i} / 2+h_{i} / 2 .
$$

The power diagram function is the upper envelope of all supporting hyper planes

$$
u(x):=\max _{i}\left\{\left\langle p_{i}, x\right\rangle-\frac{1}{2}\left\langle p_{i}, p_{i}\right\rangle+\frac{1}{2} h_{i}\right\}
$$

Hence the power diagram on $P$ corresponds, by vertical projection, to the graph of $u(x)$.

Optimal Transport Problem Suppose $\Omega$ is a domain in $\mathbb{R}^{n}$ and $\mu$ a Borel measure with $\mu\left(\mathbb{R}^{n}\right)$ being the total volume of $\Omega$. Consider transport maps $T:(\Omega, d x) \rightarrow\left(\mathbb{R}^{n}, \mu\right)$ which are measure preserving, $T^{*} \mu=d x$. The cost for the mapping is defined as

$$
C(T):=\int_{\Omega}|x-T(x)|^{2} d x .
$$

In Brenier's seminal work [8], he proved the following fundamental theorem,

Theorem 2.1 (Brenier). Let $\Omega$ be an n-dimensional compact convex set in $\mathbb{R}^{n}$ and $\mu$ any Borel measure on $\mathbb{R}^{n}$, so that $\mu\left(\mathbb{R}^{n}\right)$ is the volume of $\Omega$. Then there exists a convex function $u$ on $\Omega$, unique up to adding a constant, so that the gradient map

$$
\nabla u:(\Omega, d x) \rightarrow\left(\mathbb{R}^{n}, \mu\right)
$$

is measure preserving and $\nabla u$ minimizes the quadratic cost $\int_{\Omega}|x-T(x)|^{2} d x$ among all transport maps $T$.

In the following, we call the convex function $u$ the Brenier potential, and the gradient map $\nabla u$ the Brenier map.

In the discrete setting for optimal transport problem, we take the measure $\mu$ with finite support, i.e. $\mu=$ $\sum_{i=1}^{k} w_{i} \delta_{p_{i}}$, where $w_{i}>0$ and $\delta_{p}$ is the Dirac measure. Then the discrete Brenier theorem is as follows:

Corollary 2.2. Let $\Omega$ be an n-dimensional compact convex set in $\mathbb{R}^{n}$, point set $P=\left\{p_{1}, p_{2}, \cdots, p_{k}\right\} \subset \mathbb{R}^{n}$, with weights $w_{1}, w_{2}, \cdots, w_{k}>0$, so that $\sum_{i=1}^{k} w_{i}=$ $\operatorname{vol}(\Omega)$. Then there exists a piecewise linear convex function $u: \Omega \rightarrow \mathbb{R}$, so that $\Omega$ is decomposed to $k$ convex sets $W_{1}, W_{2}, \cdots, W_{k}$ with the property that

1. $\left.u\right|_{W_{i}}$ is linear with $\left.\nabla u\right|_{W_{i}}=p_{i}$,

2. Area $\left(W_{i}\right)=w_{i}$ for all $i$.

so that $\nabla u$ is the solution to optimal transport problem for $\Omega$ and $\left\{\left(p_{1}, w_{1}\right), \cdots,\left(p_{k}, w_{k}\right)\right\}$ with quadratic cost. The convex function u is unique up to a constant.

Variational Principle for Finite Brenier Map We have found a finite dimensional variational principle for constructing the finite Brenier map. Fix a finite point set $P=$ $\left\{p_{1}, p_{2}, \cdots, p_{k}\right\}$, the powers are $\mathbf{h}=\left(h_{1}, h_{2}, \cdots, h_{k}\right)$, the power diagram of $\left\{\left(p_{i}, h_{i}\right)\right\}$ in $\mathbb{R}^{n}$ partitions $\Omega$ to cells $\left\{W_{1}, W_{2}, \cdots, W_{k}\right\}$, the areas are $\mathbf{w}=\left(w_{1}, w_{2}, \cdots, w_{k}\right)$. 
Then the power diagram function $u(x)$ Eqn.3 is the Brenier potential, the gradient map is the Brenier map $\nabla u$ : $W_{i} \rightarrow p_{i}$, which minimizes the quadratic distance cost $C(T)=\int_{\Omega}|x-T(x)|^{2} d x$ for all the maps with the measure preserving property

$$
\operatorname{Vol}\left(T^{-1}\left(p_{i}\right)\right)=\operatorname{Vol}\left(W_{i}\right)=w_{i}, i=1,2, \cdots, k .
$$

Furthermore, we treat the areas $\mathbf{w}$ as the function of the powers $\mathbf{h}$, then the mapping $\mathbf{h} \rightarrow \mathbf{w}$ is a diffeomorphism. Let $W:=\left\{\mathbf{w} \mid \sum_{i} w_{i}=\operatorname{vol}(\Omega), w_{i}>0\right\}$ be the space of all possible area vectors, and $H:=\left\{\mathbf{h} \mid \sum_{i} h_{i}=0, \forall w_{i}>\right.$ $0\}$ be the space of all possible power vectors, then

Theorem 2.3 (Main Theorem). Let $\Omega$ be a convex domain in $\mathbb{R}^{n}$. Fix the point set $P$, given a power vector $\mathbf{h} \in H$, let $\mathrm{w}$ be the area vector associated to the convex cell decomposition of $\Omega$ induced by the power diagram for $\left\{\left(p_{i}, h_{i}\right)\right\}$, then the mapping $\mathbf{w}=\phi(\mathbf{h}): H \rightarrow W$ is a diffeomorphism.

Proof. We prove the theorem for dimension 2, which can be generalized to arbitrary dimension straightforwardly.

Let the power diagram for $\mathbf{h}$ be $D_{\mathbf{h}}$, the dual Power Delaunay triangulation be $T_{\mathbf{h}}$. Any edge $\bar{e} \in D_{\mathbf{h}}$ has a unique dual edge $e \in T_{\mathbf{h}}$. Suppose two Voronoi cells $W_{i}$ and $W_{j}$ shares an edge $\bar{e}_{i j}$, the direct computation shows

$$
\frac{\partial w_{i}}{\partial h_{j}}=\frac{\partial w_{j}}{\partial h_{i}}=\frac{\left|\bar{e}_{i j}\right|}{\left|e_{i j}\right|}>0 .
$$

and

$$
\frac{\partial w_{i}}{\partial h_{i}}=-\sum_{j \neq i} \frac{\partial w_{i}}{\partial h_{j}} .
$$

We construct a differential 1-form

$$
\omega=\sum_{i=1}^{k} w_{i} d h_{i}
$$

From Eqn.5, $\omega$ is closed, $d \omega=0$. From Brunn-Minkowski theorem [2], we know $H$ is a convex domain. Therefore, $\omega$ is an exact form. We then define an energy function

$$
E(\mathbf{h}):=\int^{\left(h_{1}, h_{2}, \cdots, h_{k}\right)} \sum_{i=1}^{k} w_{i} h_{i} .
$$

The Hessian matrix of $E$ is given by

$$
\frac{\partial^{2} E}{\partial h_{i} \partial h_{j}}=\frac{\partial w_{j}}{\partial h_{i}},
$$

From Eqn.5 and Eqn.6, we know the negative of the Hessian is diagonal dominant, so the Hessian is negative definite, the energy $E$ is concave. From the convexity of $H$ and the concavity of $E$, we conclude the gradient mapping

$$
\mathbf{h} \rightarrow \nabla E(\mathbf{h})=\mathbf{w}
$$

is a diffeomorphism.
In practice, the target area vector is given by $\overline{\mathbf{w}}=$ $\left(\bar{w}_{1}, \bar{w}_{2}, \cdots, \bar{w}_{k}\right)$, then Brenier map $T$ can be computed as follows. Construct the energy

$$
\bar{E}(\mathbf{h})=\sum_{i=1}^{k} \bar{w}_{i} h_{i}-\int^{\left(h_{1}, h_{2}, \cdots, h_{k}\right)} \sum_{j=1}^{k} w_{j} d h_{j},
$$

which is convex and can be minimized using Newton's method. From the minimizer $\mathbf{h}$, we construct the power Voronoi diagram $D_{\mathbf{h}}$, which partitions $\Omega$ to convex polygonal cells $\left\{W_{1}, W_{2}, \cdots, W_{k}\right\}$, and the power Voronoi diagram function $u(x)$ using Eqn.3. Then $u(x)$ is the Brenier potential, and $T=\nabla u(x)$ is the Brenier map, $T\left(W_{i}\right)=p_{i}$.

\section{Algorithm for Area Preserving Mapping}

Given a simply connected surface $(S, \mathbf{g})$ with total area $\pi$, fix an interior point $p_{0}$ and a boundary point $p_{1}$, then according to Riemann mapping theorem, there is a unique conformal mapping $\phi: S \rightarrow \mathbb{D}$, where $\mathbb{D}$ is unit disk, such that $\phi\left(p_{0}\right)=0$ and $\phi\left(p_{1}\right)=1$. The mapping $\phi$ parameterizes the surface, such that the Riemannian metric $\mathbf{g}$ can be represented by $\mathbf{g}=e^{2 \lambda}\left(d x^{2}+d y^{2}\right)$. The conformal factor defines a measure on the unit disk $\mu=e^{2 \lambda} d x d y$. Then there exists a unique Brenier mapping $\tau:(\mathbb{D}, d x d y) \rightarrow(\mathbb{D}, \mu)$. The composition mapping $\tau^{-1} \circ \phi: S \rightarrow \mathbb{D}$ is an area preserving mapping.

In practice, the surface is approximated by a triangle mesh $M$, normalized by a scaling such that the total area is $\pi$. The conformal mapping $\phi: M \rightarrow \mathbb{D}$ can be achieved using discrete Ricci flow method [34]. Then the measure $\mu$ can be defined on each vertex $v_{i} \in M$, as

$$
\mu\left(v_{i}\right):=\frac{1}{3} \sum_{j k} \operatorname{Area}\left(\left[v_{i}, v_{j}, v_{k}\right]\right),
$$

where $\left[v_{i}, v_{j}, v_{k}\right]$ is a triangle face adjacent to $v_{i}$. Then the sites are $P=\left\{\phi\left(v_{1}\right), \phi\left(v_{2}\right), \cdots, \phi\left(v_{n}\right)\right\}$. The target area vector is $\overline{\mathbf{w}}=\left\{\mu\left(v_{1}\right), \mu\left(v_{2}\right), \cdots, \mu\left(v_{n}\right)\right\}$, the power vector $\mathbf{h}=\left(h_{1}, h_{2}, \cdots\right)$ can be obtained by optimizing the convex energy Eqn.9 using Newton's method.

Initially, we set all powers to be zeros and translate and scale $P$, such that $P$ is contained in the unit disk. Compute the power diagram, calculate the areas for each cell $w_{i}$. Then compute the dual power Delaunay triangulation, compute the lengths of edges in the diagram and triangulation, form the Hessian matrix $H$ using Eqn.8, then update the power $\mathbf{h} \leftarrow \mathbf{h}+H^{-1}(\overline{\mathbf{w}}-\mathbf{w})$. Repeat this procedure until the cell areas are close to the target areas.

Then the power diagram for $\left\{\left(\phi\left(v_{i}\right), h_{i}\right\}\right.$ partitions $\mathbb{D}$ to convex polygonal cells $\left\{W_{i}\right\}$, the Brenier map is given by $\tau: W_{i} \rightarrow \phi\left(v_{i}\right)$. Compute the centroid of $W_{i}$, denoted as $c_{i}$. The area preserving mapping is given by $\tau^{-1} \circ \phi\left(v_{i}\right)=$ $c_{i}$. The algorithm details are illustrated in Alg.1. 


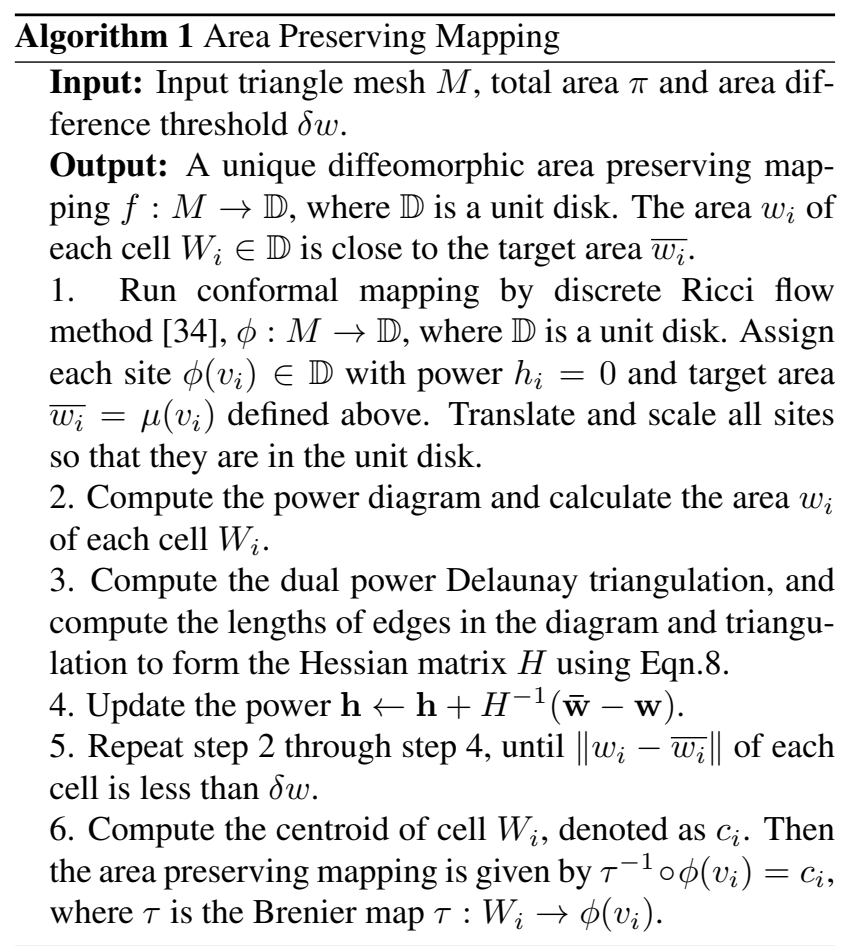

\section{Experimental Results}

We applied our area preserving mapping method to various anatomical surfaces extracted from 3D MRI scans of the brain. The baseline T1 images are acquired as part of the Alzheimer's Disease Neuroimaging Initiative (ADNI) [22]. In the paper, the segmentations are done with publicly available software FreeSurfer [13] or FIRST [29]. All surfaces are represented as triangular meshes. All experiments are implemented on laptop computer of Intel Core i7 CPU, M620 2.67GHz with 4GB memory.

\subsection{Application of Caudate Surface Parameteriza- tion}

We tested our algorithm on the left caudate nucleus surface. The caudate nucleus is a nucleus located within the basal ganglia of human brain. It is an important part of the brain's learning and memory system, for which parametric shape models were developed for tracking shape differences or longitudinal atrophy in diseases, such as Alzheimers Disease [25] and Parkinsons disease [4], etc.

Figure 2 (a) shows the triangular mesh of a reconstructed left caudate surface segmented by FIRST. The long and slim surface is challenging to compute its parametric surface. For example, a conformal mapping on slim surface usually introduces area distortions at the exponential level and may cause big numerical problems. In contrast, our method evenly embeds the caudate surface to the parametric domain and keeps the area element unchanged. For implementa- tion, we cut a small hole at the bottom of (a) to get an open boundary to make its topology consistent with a disk's. Figure 2 (b) shows that most parts of conformal mapping result shrink towards the center, while the area preserving method shown in Figure 2 (c) gives a good mapping, keeping the same area element, without much numerical error. Figure 3 are the histograms of area distortion of result surface triangles to original surface triangles for conformal mapping and area preserving mapping, respectively. It shows that conformal mapping cause up to $2^{20}$ times shrinkage, while area preserving mapping almost keep the same area. In Figure 4 , we put circle textures on both conformal mapping result and area preserving result, it gives a direct visualization of our method's correctness. Although multi-subject studies are clearly necessary, this demonstrates our area preserving method may potentially be useful to study some morphometry change to classify and compare different subcortical structure surfaces.

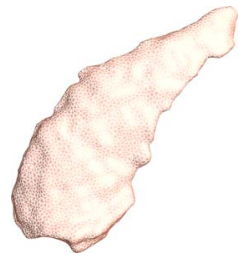

(a)

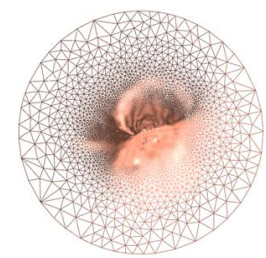

(b)

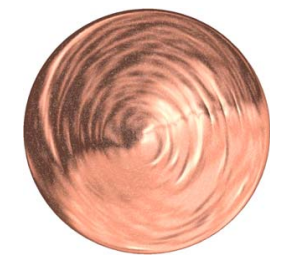

(c)
Figure 2. Comparison of geometric mappings for caudate surface: (a) original caudate surface represented by a triangular mesh; (b) conformal mapping result; (c) area preserving mapping result. The area preserving mapping method evenly maps the surface to the unit disk and eliminates the big distortions close to the upper tip area in (a).

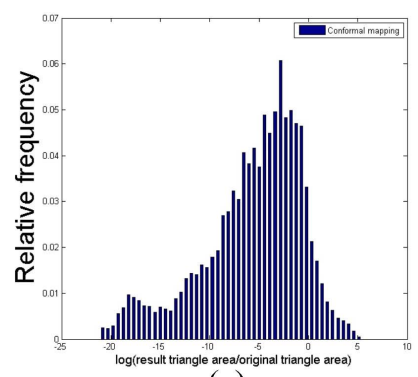

(a)

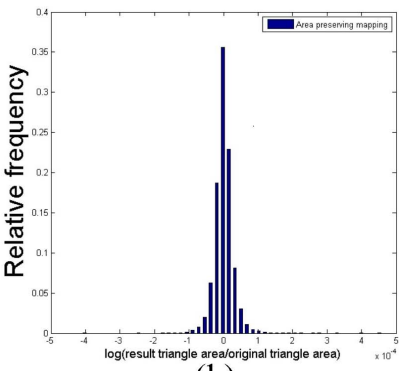

(b)
Figure 3. Histogram of area distortion: (a) area distortion of conformal mapping; (b) area distortion of area preserving mapping. The area preserving mapping result shows a much smaller area distortion.

\subsection{Application of Alzheimer's Disease Diagnosis}

For Alzheimer's disease, structural MRI measurements of brain shrinkage are one of the best established biomarkers of $\mathrm{AD}$ progression and pathology. And early researches $[30,13]$ have demonstrated that surface-based 


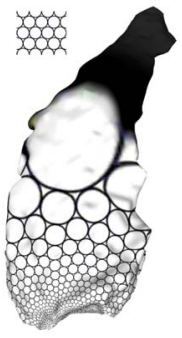

(a)

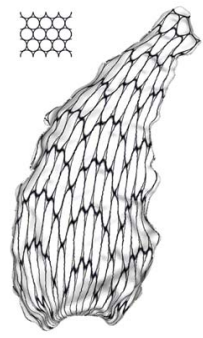

(b)
Figure 4. Circle packing of different geometric mappings: (a) circle packing of conformal mapping. (b) circle packing of area preserving mapping. The parameterizations are illustrated by the texture map of a uniformly distributed circle patterns on the caudate surface, the circle texture is shown in the upper left corner. In (a), the circles stay the circle but the circle areas change dramatically on the upper tip area. In (b), the circles become ellipses but the areas stay unchanged.

brain mapping may offer advantages over volume-based brain mapping [5] to study structural features of brain, such as cortical gray matter thickness, complexity, and patterns of brain change over time due to disease or developmental process. According to prior AD researches [15, 14], the brain atrophy is an important biomarker of AD. The atrophy may not only be area shrinkage, but also have anisotropic directions. Therefore, a good shape signature contains both area and angle deformation information may have a good potential to be a practical biomarker.

In this work, we proposed to use Beltrami coefficients [16] computed from area preserving mapping result to conformal mapping result, as a shape signature to analyze the human brain cortical surfaces among AD patients and CTL subjects. This kind of signature combines both area and angle information so that it may provide more powerful statistical ability in the AD diagnosis in the early stage.

Data Source: Our data included baseline MRI images from $50 \mathrm{AD}$ patients and 50 healthy control (CTL) subjects (Age: AD: $75.86 \pm 7.65$; CTL: $74.56 \pm 4.16$; MMSE score: AD: $22.96 \pm 2.15$; CTL: $29.02 \pm 1.04)$. We used Freesurfer's automated processing pipeline [13] for automatic skull stripping, tissue classification, cortical surface extraction, vertex correspondences across brain surfaces and cortical parcellations. According to work [11], we labeled the functional areas of a left brain cortical surface shown in Figure 5 (a) and (b).

\subsubsection{Cortical Surface Parameterization Results}

Figure 5 (c)-(f) are the conformal mapping results and area preserving mapping results of the left brain cortical surfaces of a healthy control subject and an AD patient. On both surfaces, we cut a hole around the unlabeled subcortical region [11]. After the cutting, the remaining cortical sur- face becomes a genus zero surface with one open boundary. Both algorithms compute a diffeomorphism map between the cortical surface and a unit disk. The results show that the conformal mapping results have much more area distortion on the areas close to the boundary while the area preserving mapping provides a map which preserves the area of each individual functional area. The area preserving mapping has a potential to better visualize certain sulci areas which are deeply buried under gyri, and hence to provide a tool for a more accurate manual landmark delineation operation.

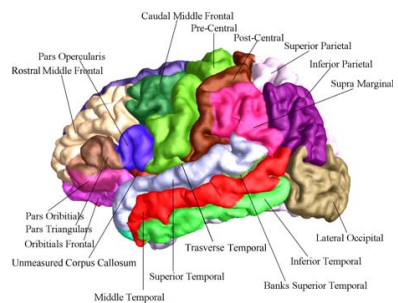

(a)

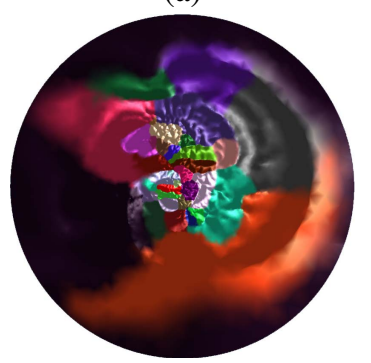

(c)

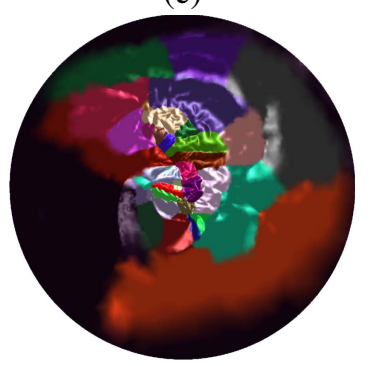

(e)

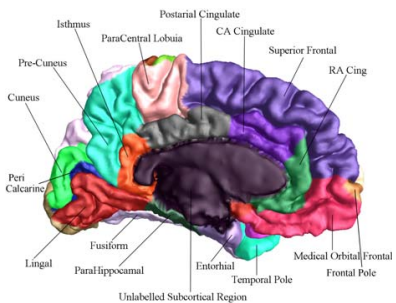

(b)

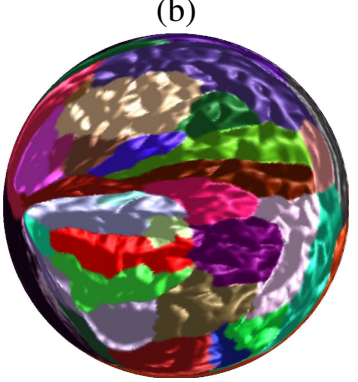

(d)

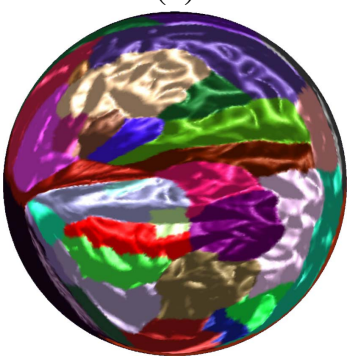

(f)
Figure 5. (a) and (b) illustrate the functional areas on the left brain cortex [11]. (a) Lateral view. (b) Medial view. (c) and (e) are conformal mapping results of a CTL subject and an AD patient, respectively; (d) and (f) are area preserving mapping results of a CTL subject and an AD patient, respectively. The area preserving mapping may provide a better visualization tool for tracking sulci landmark curves on cortical surfaces.

\subsubsection{Numerical Analysis of Signatures among Healthy Control Subjects and AD Patients}

The Beltrami coefficient is a complex-valued function defined on surfaces with supreme norm strictly less than 1 . It measures the local conformality distortion of surface maps. We tested the discrimination ability of our shape signature 
on a set of left and right brain surfaces of 50 CTL subjects and 50 AD patients. Previous work [28] indicated ten functional areas having significant atrophy in AD group, such as Middle Temporal, Superior Temporal, etc. Among the 35 functional areas, we chose 3 regions for study, which are Middle Temporal, Superior Temporal and Fusiform as shown in Figure 5 (a) and (b). Figure 6 shows the average histograms of the norm of Beltrami coefficients of $50 \mathrm{AD}$ patients and 50 CTL subjects on these three functional areas. The histograms show the norm of Beltrami coefficients of cortical surfaces of AD patients are obviously larger than those of healthy control subjects. It means that AD patients may have larger conformality distortion in both area and shrinkage directions because AD patients may suffer a more serious atrophy of brain structures which result from a combination of neuronal atrophy, cell loss and impairments in myelin turnover and maintenance [14].

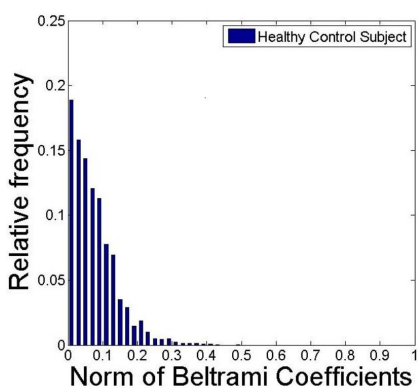

(a)

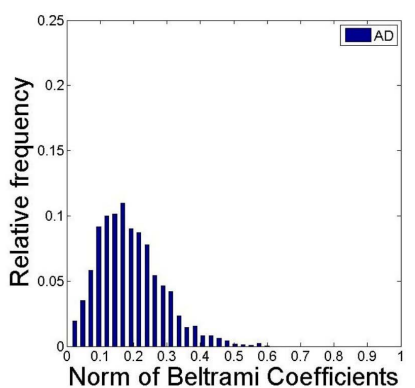

(b)
Figure 6. Histograms of Norm of Beltrami Coefficients: (a) result of healthy control subjects. (b) result of AD patients. The AD result demonstrated a stronger and more anisotropic deformation due to a more serious atrophy of brain structures.

\subsubsection{Classification among Healthy Control Subjects and AD Patients}

We further hypothesize that the our computed Beltrami coefficients may help early AD detection. We performed the classification between AD and CTL groups in the current ADNI dataset. For the classification experiment, $80 \%$ of each category of both left and right brain cortical surfaces are set to be training samples and the remaining $20 \%$ as testing samples. To obtain fair results, we randomly selected the training set each time and computed the average recognition rate over 1000 times. We used Support Vector Machine (SVM) [1] as a classifier, where the linear kernel function was employed, and we used $C$-SVM and chose $C=5$ by running cross validation. Table 1 shows 95 percent confidence interval for average recognition rate of our method is $87.50 \% \pm 0.55 \%$. For comparisons, we also computed area based method and volume based method. For area based method, we computed the surface areas for the base domain and 3 regions mentioned above on each hemisphere as a sig-

\begin{tabular}{|l|c|}
\hline Method & Rate $\%$ \\
\hline \hline Area & $70.00 \% \pm 0.73 \%$ \\
Volume & $62.50 \% \pm 0.57 \%$ \\
Our method & $87.50 \% \pm 0.55 \%$ \\
\hline
\end{tabular}

Table 1. Average recognition rate(\%) for applying different signatures among 50 healthy control subjects and $50 \mathrm{AD}$ patients. In the experiments, $80 \%$ data are used for training and the remaining for testing. The experiments were repeated over 1000 times and 95\% confidence intervals are reported here.

nature $($ Area $)=\left(A_{0}, A_{1}, A_{2}, A_{3}\right) ; 95$ percent confidence interval for the average recognition rate is $70.00 \% \pm 0.73 \%$. We also calculated the volume of each hemisphere as a signature $(\mathrm{Vol}), 95$ percent confidence interval for the average recognition rate is $62.50 \% \pm 0.57 \%$. Although the above two methods are not popular signatures for AD study in the literature and a more careful study such as [10] is necessary, the results helped illustrate the various nature of our testing data and showed the potential of our proposed shape signature.

\section{Conclusions and Future Work}

In this paper, we presented a method to compute area preserving mapping between surfaces based on Brenier's approach in Optimal Mass Transport theory. Our approach produces the unique diffeomorphic mapping. Comparing to the existing method, our method improves the simplicity and efficiency by significantly reducing the complexity (from $n^{2}$ unknowns to $n$ ). Therefore, our method offers a stable and effective way to compute area preserving mapping in $2 D$ parametric coordinates. Our experimental results show our work may provide novel ways for shape analysis and improve the statistical power for detecting abnormalities in brain surface morphology. In the future, we will explore and validate other broad shape analysis applications in medical imaging research.

\section{References}

[1] http://www.csie.ntu.edu.tw/ cjlin/libsvm/.

[2] A. D. Alexandrov. Convex Polyhedra. Springer, 2005.

[3] S. Angenent, S. Haker, A. Tannenbaum, and R. Kikinis. Conformal geometry and brain flattening. In Med. Image Comput. Comput.Assist. Intervention, pages 271-278, 1999.

[4] L. G. Apostolova, M. Beyer, A. E. Green, K. S. Hwang, J. H. Morra, Y. Y. Chou, C. Avedissian, D. Aarsland, C. C. Janvin, J. L. Cummings, and P. M. Thompson. Hippocampal, caudate, and ventricular changes in Parkinson's disease with and without dementia. Mov. Disord., pages 687-695, 2010.

[5] J. Ashburner, C. Hutton, R. Frackowiak, I. Johnsrude, C. Price, and K. Friston. Identifying global anatomical differences: deformation-based morphometry. Human Brain Mapping, 6(5-6):348-357, 1998. 
[6] M. Balasubramanian, J. Polimeni, and E. Schwartz. Exact geodesics and shortest paths on polyhedral surfaces. IEEE Trans. Patt. Anal. Mach. Intell., pages 1006-1016, 2009.

[7] D. M. Boyer, Y. Lipman, E. St Clair, J. Puente, B. A. Patel, T. Funkhouser, J. Jernvall, and I. Daubechies. Algorithms to automatically quantify the geometric similarity of anatomical surfaces. Proc. Natl. Acad. Sci., 108:18221-18226, 2011.

[8] Y. Brenier. Polar factorization and monotone rearrangement of vector-valued functions. Com. Pure Appl. Math., 64:375417, 1991.

[9] A. M. Bronstein, M. M. Bronstein, and R. Kimmel. Generalized multidimensional scaling: a framework for isometryinvariant partial surface matching. Proc. Natl. Acad. Sci., 103:1168-1172, 2006.

[10] R. Cuingnet, E. Gerardin, J. Tessieras, G. Auzias, S. Lehericy, M. Habert, M. Chupin, H. Benali, , and O. Colliot. Automatic classification of patients with Alzheimer's disease from structural MRI: A comparison of ten methods using the ADNI database. Neuroimage, 56(2), 2011.

[11] R. S. Desikan, F. Segonne, B. Fischl, B. T. Quinn, B. C. Dickerson, D. Blacker, R. L. Buckner, A. M. Dale, R. P. Maguire, B. T. Hy-man, M. S. Albert, and R. J. Killiany. An automated labelingsystem for subdividing the human cerebral cortex on MRI scans into gyral based regions of interest. Neuroimage, 31:968-980, 2006.

[12] H. A. Drury, D. C. Van Essen, C. H. Anderson, C. W. Lee, T. A. Coogan, and J. W. Lewis. Computerized mappings of the cerebral cortex: A multiresolution flattening method and a surface-based coordinate system. J. Cognitive Neurosciences, 8:1-28, 1996.

[13] B. Fischl, M. I. Sereno, and A. M. Dale. Cortical surfacebased analysis II: Inflation, flattening, and a surface-based coordinate system. NeuroImage, 9(2):195 - 207, 1999.

[14] N. C. Fox, R. I. Scahill, W. R. Crum, and M. N. Rossor. Correlation between rates of brain atrophy and cognitive decline in AD. Neurology, 52:1687-1689, 1999.

[15] G. B. Frisoni, N. C. Fox, C. R. J. Jr, P. Scheltens, and P. M. Thompson. The clinical use of structural MRI in Alzheimer's disease. Nature Reviews Neurology, 6(2):67-77, 2010.

[16] F. Gardiner and N. Lakic. Quasiconformal teichmuller theory. American Mathematics Society, 2000.

[17] X. Gu, Y. Wang, T. F. Chan, P. M. Thompson, and S.-T. Yau. Genus zero surface conformal mapping and its application to brain surface mapping. IEEE Trans. Med. Imag., 23:949958, 2004

[18] X. Gu and S.-T. Yau. Computing conformal structures of surfaces. Communications in Information and Systems, 2:121146, 2002.

[19] S. Haker, L. Zhu, A. Tannenbaum, and S. Angenent. Optimal mass transport for registration and warping. International Journal on Computer Vision, 60(3):225-240, 2004.

[20] R. S. Hamilton. The Ricci flow on surfaces. Mathematics and general relativity, 71:237-262, 1988.

[21] M. K. Hurdal and K. Stephenson. Cortical cartography using the discrete conformal approach of circle packings. NeuroImage, 23:S119-S128, 2004.
[22] C. R. J. Jack, M. A. Bernstein, N. C. Fox, P. M. Thompson, G. Alexander, D. Harvey, B. Borowski, P. J. Britson, J. L. Whitwell, C. Ward, and et al. The Alzheimer's disease neuroimaging initiative (ADNI): MRI methods. J. of Mag. Res. Ima., 27:685-691, 2007.

[23] L. Ju, M. K. Hurdal, J. Stern, K. Rehm, K. Schaper, and D. Rottenberg. Quantitative evaluation of three surface flattening methods. NeuroImage, 28:869-880, 2005.

[24] L. V. Kantorovich. On a problem of monge. Uspekhi Mat. Nauk., 3:225-226, 1948.

[25] S. K. Madsen, A. J. Ho, X. Hua, P. S. Saharan, A. W. Toga, C. R. Jack, M. W. Weiner, and P. M. Thompson. 3D maps localize caudate nucleus atrophy in 400 Alzheimer's disease, mild cognitive impairment, and healthy elderly subjects. Neurobiol. Aging, 31:1312-1325, 2010.

[26] T. Rehman, E. Haber, G. Pryor, and A. Tannenbaum. Fast optimal mass transport for 2D image registration and morphing. Elsevier Journal of Image and Vision Computing, 2008.

[27] E. Sharon and D. Mumford. 2D-shape analysis using conformal mapping. In Proc. IEEE Conf. Computer Vision and Pattern Recognition, pages 350-357, 2004.

[28] Y. Shi, R. Lai, and A. Toga. Corporate: cortical reconstruction by pruning outliers with Reeb analysis and topologypreserving evolution. Information Process Medical Imaging, 22:233-244, 2011.

[29] S. M. Smith, M. Jenkinson, M. W. Woolrich, C. F. Beckmann, T. E. Behrens, H. Johansen-Berg, P. R. Bannister, M. De Luca, I. Drobnjak, D. E. Flitney, R. K. Niazy, J. Saunders, J. Vickers, Y. Zhang, N. De Stefano, J. M. Brady, and P. M. Matthews. Advances in functional and structural MR image analysis and implementation as FSL. Neuroimage, 23 Suppl 1:S208-219, 2004.

[30] P. M. Thompson and A. W. Toga. A surface-based technique for warping 3-dimensional images of the brain. IEEE Trans. Med. Imag., 15:1-16, 1996.

[31] B. Timsari and R. M. Leahy. Optimization method for creating semi-isometric flat maps of the cerebral cortex. Medical Imaging 2000: Image Processing, 3979:698-708, 2000.

[32] D. Tosun and J. Prince. A geometry-driven optical flow warping for spatial normalization of cortical surfaces. IEEE Trans. Med. Imag., 27:1739-1753, 2008.

[33] Y. Wang, X. Gu, T. F. Chan, P. M. Thompson, and S.-T. Yau. Conformal slit mapping and its applications to brain surface parameterization. In Med. Image Comp. Comput.-Assist. Intervention, Proceedings, Part I, pages 585-593, 2008.

[34] Y. Wang, J. Shi, X. Yin, X. Gu, T. F. Chan, S. T. Yau, A. W. Toga, and P. M. Thompson. Brain surface conformal parameterization with the Ricci flow. IEEE Trans Med Imaging, 31(2):251-264, 2012.

[35] W. Zeng, X. Yin, Y. Zeng, Y. L. X. Gu, and D. Samaras. 3D face matching and registration based on hyperbolic Ricci flow. CVPR Workshop on 3D Face Processing, pages 1-8, 2008.

[36] L. Zhu, S. Haker, and A. Tannenbaum. Area-preserving mappings for the visualization of medical structures. In Medical Image Computing and Computer-Assisted Intervention, 2879:277-284, 2003. 